\title{
CORRESPONDENCE
}

\section{Don't release other people's data without their consent}

SIR - I am astounded by the audacity of someone photographing the presentation of another researcher and then publishing their data without the presenter's permission ('Physicists aflutter about data photographed at conference' Nature 455, 7; 2008). In what scientific forum, other than apparently the arXiv.org preprint server, is that permissible practice?

The implication in your News story that videorecording conference proceedings somehow justifies this unfortunate incident is misleading. The point is not that data are recorded through a particular medium. We all take notes at meetings in some form, whether physical or mental, and who hasn't had their poster transcribed nearly verbatim? Rather, the issue is whether the information is released in a fair and representative manner.

Although, as you note, videotaping conference proceedings is common in biology, we operate under an implicit, and often explicit, ethic that data presented at meetings are personal communications. As such, publication of personal communications by a second party requires formal approval from the originating researchers. This practice strikes a balance between the public good that arises from collegial sharing of preliminary results and preservation of investigators' rights to ownership of their intellectual work. Therefore, except under exceptional circumstances, scientists ought to obtain permission to cite the unpublished works of others. Sometimes investigators may unfairly withhold data that are so critical that they justify overlooking what is the norm in most academic communities. But any such putatively exceptional case of data release should

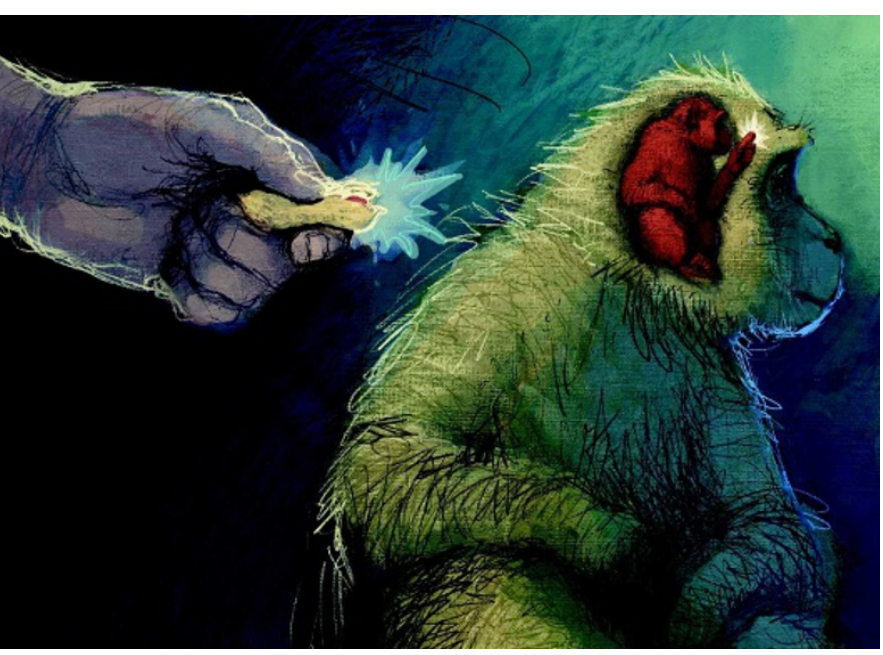

require unequivocal justification.

Ethics aside, what exactly was the purpose of reporting incompletely vetted, possibly erroneous experimental results? Can the group who released the data provide assurance that the information gleaned during the presentation adequately represents the original data in all its potential complexity? If not, there seems to be little justification for, or value in, usurping the intellectual rights of the group that originally generated and presented the results. And it is doubtful whether all those who contributed to the project received proper credit.

This case violates the spirit of collegiality that most scientists hold as an ideal in our public discourse. We all accept that others may scoop our work. We should not have to worry about being scooped by our own data.

Daniel N. Frank Molecular, Cellular and Developmental Biology, Mucosal and Vaccine Research Program Colorado, University of Colorado-Boulder, Boulder, Colorado 80309, USA e-mail:daniel.frank@colorado.edu

\section{Further reflections on how we interpret the actions of others}

SIR - In their Essay 'Behind the looking-glass' (Nature 454, 167-168; 2008), Antonio Damasio and Kaspar Meyer suggest how mirror neurons might work. But they need to reflect on other aspects of the mirror phenomenon to complete the picture.

Mirror neurons are known for their intriguing property of discharging when a particular motor act is either being performed or being observed. Damasio and Meyer describe them as neural ensembles in higher-order association areas called CDZs (for 'convergencedivergence zones') that collect information from specific sensory areas and signal back to those areas. Action understanding (as in the authors' example of hearing a peanut being cracked) depends on activation of this network.

The CDZ model attempts to explain the mechanism underlying action understanding. But it overlooks a fundamental feature of the mirror mechanism: that is, the capacity to transform sensory information into a motor format - why should we have a copy of the actions of others in our motor system?

We can certainly recognize biological actions using sensory information and performing the kind of processing suggested by the CDZ model. But mirror neurons indicate that we must also have another mechanism for understanding another's actions. That mechanism directly maps sensory information on cortical motor neurons, providing the observer with an immediate representation of the motor acts being performed by others. There is no need for a higher-order association, as the CDZ model requires.

This, of course, does not imply that mirror neurons alone 'understand' the actions of others. Such an interpretation of the mirror system would go against all we know about the complexity of cortical organization. The point at issue is the specific contribution of mirror neurons to action understanding. Because of their motor nature, these neurons add a new, personal dimension to our capacity for understanding others that is based on our own motor knowledge and experience.

So, in spite of its heuristic value, the CDZ model underestimates the motor aspect of the mirror mechanism. It was this mechanism that prompted the description of action understanding as "the result of a 'first-person' process where the self feels like an actor, rather than a spectator" (M. Jeannerod The Cognitive Neuroscience of Action, Blackwell, 1997).

Giacomo Rizzolatti Dipartimento di Neuroscienze, Università di Parma, Via Volturno 39, 43100 Parma, Italy e-mail: giacomo.rizzolatti@unipr.it Corrado Sinigaglia Dipartimento di Filosofia, Università degli Studi di Milano, Via Festa del Perdono 7, 20122 Milano, Italy

\section{Austria: Academy of Sciences states its case}

SIR - As president and secretarygeneral of the Austrian Academy of Sciences, we wish to clarify the academy's position in the investigation into the alleged scientific misconduct associated with the urological clinical trial that you discuss in your Editorial 'Scandalous behaviour' (Nature 454, 917-918; 2008). Contrary to your implications, the academy is committed to help in the resolution of this case.

You note that the academy put 